Meta

Journal des tradlucteurs

Translators' Journal

\title{
Étude onomasiologique d'un vocabulaire politique : le vocabulaire européen
}

\section{Nestor Schumacher}

Volume 19, numéro 4, décembre 1974

URI : https://id.erudit.org/iderudit/004057ar

DOI : https://doi.org/10.7202/004057ar

Aller au sommaire du numéro

Éditeur(s)

Les Presses de l'Université de Montréal

ISSN

0026-0452 (imprimé)

1492-1421 (numérique)

Découvrir la revue

Citer cet article

Schumacher, N. (1974). Étude onomasiologique d'un vocabulaire politique : le vocabulaire européen. Meta, 19(4), 197-202. https://doi.org/10.7202/004057ar d'utilisation que vous pouvez consulter en ligne. 


\section{Étude onomasiologique d'un vocabulaire politique: le vocabulaire européen}

Depuis que le phénomène complexe qu'il est convenu d'appeler l'intégration européenne a commencé à se manifester concrètement aux environs de l'année 1950 , on constate l'apparition progressive d'un vocabulaire spécifique, dit parfois «vocabulaire européen ». L'expression consacrée en français, la «terminologie européenne », est quelque peu abusive et moins exacte assurément que la dénomination allemande « europäischer Sprachgebrauch », laquelle met l'accent sur l'usage et inclut la phraséologie. Cette terminologie et cette phraséologie, nous les trouvons pour une part dans les documents officiels qui jalonnent la route de l'intégration européenne, mais également dans les écrits des publicistes et les articles de la presse quotidienne.

Le point de vue onomasiologique s'impose à qui veut étudier la fixation d'un tel vocabulaire. Sous l'impulsion d'un phénomène politique nouveau - ou qui du moins s'accélère nettement après la Deuxième Guerre mondiale - de nouveaux concepts se font jour, de nouvelles institutions sont créées ou projetées. Comment ces concepts, ces institutions, leurs buts et leurs moyens vont-ils être exprimés dans les langues intéressées et quels sont les phénomènes linguistiques que l'on peut observer au cours de cette période de fixation d'un vocabulaire donné, tel est en gros le sujet de l'ouvrage que nous avons consacré à cette question ${ }^{1}$.

Notre étude se limite aux deux langues qui nous sont les plus familières, le français, langue de négociation, dans laquelle le terme s'est souvent fixé d'abord, et l'allemand, langue riche en néologismes. Il serait évidemment fort souhaitable qu'une équipe de chercheurs s'attache à l'étude de l'apparition du vocabulaire européen dans les différentes langues de la Communauté.

1. En partant, non de mots individuels, mais d'une situation où quelque chose est à créer et à dénommer, nous constatons d'abord - comme d'autres chercheurs

1. En préparation : Der Wortschatz der europäischen Integration, Band ... der Reihe "Sprache der Gegenwart ", Schriften des Instituts für deutsche Sprache, Mannheim (Pädagogischer Verlag Schwann, Düsseldorf). 
dans des domaines différents - le plus souvent une sorte de flottement, une série de tâtonnements, successifs ou concomittants, phénomène qui souligne la richesse des ressources créatrices de la langue.

a) Il se peut tout d'abord que le signifié lui-même ne soit pas encore exactement fixé et ne se décante que par la confrontation des conceptions. C'est ainsi que, au cours des négociations sur le Plan Schuman, à Paris pendant l'été 1950, le grand marché à créer — la déclaration Schuman parlait de "fusion des marchés 》 - fut d'abord appelé marché unique, puis simultanément marché unique et marché commun, jusqu'à ce que s'établisse une distinction théorique : dans un marché «unique», l'écart des prix ne serait pas supérieur aux frais de transport, dans un «marché commun », seuls les obstacles artificiels seraient supprimés de sorte que les écarts de prix exprimeraient aussi ce que les économistes appellent les avantages de la localisation. Les expressions allemandes sont dans le premier cas : einheitlicher Markt avec des variantes occasionnelles comme : einziger Markt, Einheitsmarkt, unitarischer Markt et geeinter Markt, vereinter Markt (marché unifié), dans le second : gemeinsamer Markt avec des variantes soulignant différents aspects d'une même réalité : erweiterter Markt (marché élargi), freier Markt (marché libre), kommunizierender Markt (marchés communiquants) et gemeinschaftlicher Markt (marché communautaire).

b) On aura déjà remarqué par ce seul exemple que les termes concurrents ne sont pas nécessairement des synonymes, mais qu'ils expriment l'un par rapport à l'autre une différence de perspective. Des termes assez éloignés l'un de l'autre peuvent entrer en concurrence comme le montre l'exemple de la dénomination de l'organisation globale projetée par le Plan Schuman.

Dans la première phase de négociations, on s'en tint à des termes généraux comme l'organisation (die Organisation), le complexe (das Gebilde, der Komplex, der Wirtschaftskomplex, et même das Ganze, das System) par opposition à la seule institution nommément désignée, la Haute Autorité ; les commentateurs se servent parfois de termes comme le consortium, le combinat (das Montan-Kombinat), mais 1'expression la plus usitée est sans conteste le pool (der Pool), avec des formules comme le pool charbon-acier (der Kohle- und Stahlpool), le pool Schuman (der Schuman-pool), le pool minier et sidérurgique, le pool carbo-sidérurgique et même le pool noir. Ces termes étaient empruntés au langage économique.

Pendant les négociations apparaît, tout d'abord pour désigner l'ensemble des pays participants, l'expression : la Communauté (die Gemeinschaft), on parle par exemple des «frontières de la Communauté ». Lorsqu'il fallut donner un nom à l'organisation d'ensemble dont les organes allaient être la Haute Autorité, le Conseil des Ministres, l'Assemblée et la Cour de Justice, le délégué allemand M. Ophüls proposa ce terme de Gemeinschaft (Communauté), qui se situait à un autre niveau que les termes économiques, qui rappelait la «mise en commun 》 des industries du charbon et de l'acier proposée par la France et qui de surcroît ne préjugeait pas du lien politique qui pourrait se créer entre ces pays. On sait que le terme ne figure pas seulement dans le nom de la C.E.C A., mais a été repris pour la C.E.D., la C.P.E. - toutes deux avortées - enfin pour la C.E.E. et la C.E.E.A., introduisant une catégorie nouvelle en droit international. 
c) Ces tâtonnements ne sont pas seulement successifs, mais largement concomittants. Le résultat peut être la coexistence de plusieurs termes parallèles, qui correspondent en quelque sorte à des niveaux de langue.

C'est ainsi que l'on trouve une dénomination officielle : la Communauté européenne du Charbon et de l'Acier (die Europäische Gemeinschaft für Kohle und Stahl), avec l'abréviation intégrée en français : la C.E.C.A. - lettrique en allemand : die EGKS, et une appellation plus populaire : le pool charbon-acier / die Montanunion/Montangemeinschaft (montan signifiant : qui concerne le charbon et le fer, comme dans Montanindustrie).

d) Enfin, il convient de faire la part de l'élément traduction dans le flottement, car les textes de base ont été d'abord rédigés en français et par ailleurs, la seule version officielle du Traité instituant la C.E.C.A. est la version française. Haute Autorité par exemple avait été choisi par le petit groupe entourant Jean Monnet, le véritable père du Plan Schuman; il n'y a pas d'autre appellation en français, mais les premiers documents allemands parlent de : Oberbehörde, Oberste Behörde, Oberste Autorität, ensuite uniquement de Hohe Behörde, qui avait été adopté officiellement.

2. On peut se poser la question des motifs qui expliquent un choix donné. Pour le vocabulaire institutionnel, dont relèvent les exemples cités jusqu'ici, il y a évidemment fixation officielle, mais celle-ci repose en fait sur les mêmes motifs qui déterminent la fixation spontanée de tel terme politique.

Les plus apparents sont :

a) la valeur de programme que l'on peut attribuer au terme. Il est évident que les promoteurs de l'unité européenne ont voulu souligner l'idée de solidarité, qui s'exprime notamment dans les termes de Communauté et de Marché commun.

Un autre exemple typique de mot-programme est supranational (all. supranational, übernational, überstaatlich). Le mot avait en fait été créé par des politologues français après la première guerre mondiale, mais en 1948-1950 il apparaît comme quelque chose de neuf. Pour Schuman, Adenauer, de Gasperi, partisans d'une Haute Autorité située au-dessus des États dans un secteur particulier, le terme avait valeur de programme. Il eut d'ailleurs un succès foudroyant parce qu'il distingue la nouvelle institution des organisations internationales classiques, il fait son entrée dans les Traités (C.E.C.A., C.E.D., projet de Statut de la Communauté Politique Européenne, 1953). Mais après l'échec de la Communauté Européenne de Défense, l'idée de supranationalité est de plus en plus controversée et le terme perd sa consistance. Les Présidents Hallstein et Rey notamment conseillent de le remplacer par communautaire (gemeinschaftlich).

b) Un autre élément - qui n'exclut d'ailleurs pas le premier — est le rôle joué dans la fixation des termes par certaines personnalités importantes. L'influence des négociateurs est évidente, ils écrivent sur une page blanche; ensuite interviennent les «eurocrates» qui développent la terminologie interne, enfin certains éléments extérieurs (politiciens, juristes, politologues, journalistes) diffusent le vocabulaire officiel et $\mathrm{y}$ ajoutent certains néologismes. 
Ainsi, le terme supranational paraissant inadéquat à beaucoup de juristes, P. Reuter et F. Carboni ont proposé extranational (aussernational, ausserstaatlich), F. Rosenstiel métanational (meta-national), G. Erler transnational (transnational), qui commence à percer. Politiquement, ces termes correspondent à une position de repli : on abandonne la conception d'une autorité située au-dessus des nations (supra), le nouveau pouvoir se situe «en dehors des nations 》 (extra), il s'exerce à travers les frontières nationales (trans), pour certains il n'est que le prolongement des pouvoirs nationaux (meta).

c) Il convient de rappeler aussi qu'il s'agit ici d'un phénomène international par définition, qui intéresse plusieurs langues. Il est assez naturel qu'une préférence aille aux internationalismes et aux formules paralleles. Il s'agit rarement d'emprunts nouveaux, mais la fixation de la terminologie en français entraîne généralement une fixation parallèle dans les autres langues intéressées. L'inverse se produit parfois comme dans le cas de l'Assemblée Parlementaire Européenne, rebaptisée Parlement européen le 30 mars 1962 d'après Europäisches Parlament.

d) Ajoutons enfin que dans des domaines plus techniques, un souci de précision explique certains choix. Ainsi les ressources de la C.E.C.A. sont assurées par un droit perçu sur les livraisons de charbon et d'acier. Après avoir parlé de cotisation (Beitrag), les délégués français se décidèrent pour le terme de prélèvement, qui fut rendu successivement dans les documents allemands par Erhebung, puis Entnahme et enfin Umlage, notamment dans Ausgleichs-umlage (prélèvement de péréquation). Les délégués allemands choisissaient ainsi un terme de fiscalité qui implique une répartition égale de la charge (DWB : Steuer umlegen $=$ tributum imponere civibus aequa portione). Par contre, dans le domaine du marché commun agricole, le droit d'entrée variable appelé également prélèvement a été rendu par le terme imagé de $A b s c h \ddot{p p f u n g ~(l i t t e ́ r a l e m e n t ~ « e ́ c r e ́ m a g e ~ 》) . ~}$

3. Nous avons vu qu'après une période de tâtonnement, les termes se fixaient. Parmi ces termes, certains apparaissent comme des mots clés, des pôles autour desquels gravitent les tournures stéréotypées, les composés, les dérivés, etc. Voyons comment se marque le dynamisme de ces mots clés.

a) Tout d'abord, par leur fréquence relative. Les mots à indice de fréquence les plus élevés sont : intégration - Europe - Communauté - supranational. Ceci s'explique par leur position centrale dans le système de concepts : il s'agit de dénommer le processus (intégration), l'espace à ingérer (l'Europe, potentiellement), le but (la Communauté) et le moyen (l'institution supranationale, du moins au début).

b) Les mots les plus fréquents apparaissent dans des expressions qui constituent un embryon de phraséologie : par exemple intégration partielle, sectorielle, fonctionnelle, totale, globale, générale; verticale, horizontale; politique, économique, sociale, militaire, européenne.

c) La terminologie allemande se caractérise évidemment par un foisonnement de composés, où les mots clés interviennent comme déterminant ou déterminé (respectivement 60 composés du premier type et une douzaine du second pour Integra- 
tion). Citons seulement Integrationsverdrossenheit (découragement qui s'empare des «Européens » devant les obstacles qui entravent le mouvement d'intégration) et Restintegration (intégration des secteurs non encore intégrés).

d) L'allemand forme également des dérivés avec plus de facilité, notamment à partir de composés. Citons pour Integration : Integrationist, Integrationismus, integrationistisch; integrativ, desintegrativ, reintegrativ et (un)- integrierbar; Integriertheit.

e) Le dynamisme des mots clés se répercute sur les mots de la même famille; on constate par exemple un emploi assez fréquent de : Integrierung, integrieren, sich integrieren, integriert, nicht-integriert, vollintegriert et teil-integriert. On voit resurgir certains mots peu usités comme vergemeinschaften (communautariser) et $V$ ergemeinschaftung (communautarisation) sous l'influence du sens spécifique de Gemeinschaft (la communauté).

f) Les néologismes de sens sont assez nombreux. Pensons à communautaire dans le sens «concernant la Communauté européenne» (gemeinschaftlich, Gemeinschafts-) à côté des composés intracommunautaire (innergemeinschaftlich) et extracommunautaire (aussergemeinschaftlich). Le mot Europe (Europa) prend une nouvelle dimension : l'Europe à faire, l'Europe unie. Cette nouvelle dimension se retrouve avec des nuances, dans l'adjectif européen (europäisch), les substantifs les Européens (die Europäer), européanisme ou européisme (Europäismus, Europäertum) et le verbe européaniser ou européiser (europäisieren), qui passe du sens « assimiler aux mours européennes » au sens « intégrer dans le cadre de l'Europe » ou « donner un esprit (ou un statut) européen ».

Les mots clés, pôles autour desquels gravite le vocabulaire européen, doivent être distingués des slogans, qui traversent la constellation, un peu comme des comètes. Ils se caractérisent par les traits suivants :

1) leur diffusion et éventuellement leur disparition rapides. Pensons au fameux Paneuropa (la Paneurope) du comte Coudenhove-Kalergi, slogan européen de l'entre-deux-guerres, qui a laissé des traces dans l'adjectif paneuropéen (paneuropäisch), cher à de Gaulle. On note cependant des retours réguliers : Union européenne, Fédération européenne et surtout États-Unis d'Europe se trouvent déjà dans les projets européens du $19^{\mathrm{e}}$ siècle.

2) la simplification des données : le dernier exemple cité est un calque qui masque l'énorme différence entre l'intégration américaine et la nôtre.

3) la surenchère : on sait que le Plan Schuman avait été attaqué en Allemagne par les sociaux-démocrates et la C.E.D. en France par les gaullistes et les communistes. De là une série d'appellations polémiques, procédant par ironie : par exemple Kleineuropa (la Petite Europe) est déformé en Kleinst-europa (une Mini-Europe) avec des variantes Resteuropa et Rumfeuropa (une Europe tronquée) ou par exagération: la Haute Autorité a été qualifiée à l'époque de : supercartel (Superkartell, Suprakartell, Überkartell), de supermonopole (Supermonopol, Überkonzern, Riesenkartell, Riesentrust), également de Überbehörde, Superbehörde et même de super-État (Superstaat, Suprastaat, voire Hyperstaat). 
4) par la tendance à la bipolarisation : le langage politique aime les oppositions et les crée : des termes qui ne sont pas des antonymes, mais qui marquent une différence de degré dans une même direction, deviennent, dans une situation politique donnée, deux pôles opposés. Les exemples sont nombreux : à la Petite Europe, on oppose la Grande Europe (Kleineuropa/Grosseuropa), à l'unionisme, qui se fonde sur la coopération (Unionismus), le fédéralisme, qui dans l'optique européenne a uniquement un sens centripète (Föderalismus) ; à la Fédération européenne (Europäische Föderation), qu'ils assimilent volontairement à la fusion, à l'effacement, les gaullistes opposent la Confédération européenne (Europäische Konföderation), à la Communauté politique (Politische Gemeinschaft) envisagée en 1953, on oppose 1'Union politique (Politische Union) des Plans Fouchet de 1962 , etc.

Nous avons pu voir ainsi à grands traits comment le processus de l'intégration européenne s'est exprimé dans le vocabulaire français et allemand et observer quelques constantes : flottement initial ou prolongé dans la dénomination suite à la concurrence de différents termes proches, fixation officielle pour le vocabulaire institutionnel, spontanée pour le vocabulaire politique, et due à différents facteurs, parmi lesquels la valeur de programme paraît capitale, dynamisme des mots clés, qui se marque par leur influence sur la fréquence et sur le sens spécifique des mots de la même famille, néologisme de forme (composés, dérivés) surtout en allemand, enfin récurrence de slogans simplificateurs et de termes prolémiques caractérisés par la surenchère et la tendance à la bipolarisation. Nous avons là quelques traits caractéristiques que l'on retrouverait sans doute - mutatis mutandis - dans l'étude onomasiologique de tout vocabulaire politique formant un tout cohérent.

NESTOR SCHUMACHER 